\title{
The Cranial Neural Crest in a Multiomics Era
}

\author{
Vanessa Chong-Morrison ${ }^{\dagger}$ and Tatjana Sauka-Spengler* \\ Radcliffe Department of Medicine, Weatherall Institute of Molecular Medicine, University of Oxford, Oxford, United Kingdom
}

Neural crest ontogeny plays a prominent role in craniofacial development. In this Perspective article, we discuss recent advances to the understanding of mechanisms underlying the cranial neural crest gene regulatory network (CNC-GRN) stemming from omics-based studies. We briefly summarize how parallel considerations of transcriptome, interactome, and epigenome data significantly elaborated the roles of key players derived from pre-omics era studies. Furthermore, the growing cohort of cNC multiomics data revealed contribution of the non-coding genomic landscape. As technological improvements are constantly being developed, we reflect on key questions we are poised to address by taking advantage of the unique perspective a multiomics approach has to offer.

Keywords: neural crest, multiomics, gene regulatory network, non-coding, interactome, transcriptome, epigenome

\section{INTRODUCTION}

Gene regulatory networks (GRNs) coordinate the expression of genes encoding transcription factors (TFs), cell signaling pathway components and differentiation effectors in genetic cascades mediated by cis-regulatory elements (Levine and Davidson, 2005). GRNs present a unique perspective in the understanding of developmental pathways and mechanisms by focusing on the regulated activity of genes within a defined cellular context. The lengthy process of neural crest $(\mathrm{NC})$ development, that starts at the end of gastrulation and proceeds into late organogenesis has been proposed to be orchestrated by a multi-module GRN (SaukaSpengler and Bronner-Fraser, 2008; Simões-Costa and Bronner, 2015). Broadly-speaking, Wnt, Fgf, and Bmp signals at the neural plate border activate expression of genes from the Msx, Pax, and Zic families during NC induction (Ikeya et al., 1997; LaBonne and Bronner-Fraser, 1998; Monsoro-Burq et al., 2003, 2005; Lewis et al., 2004; Schumacher et al., 2011). Pax3 and Zicl activate expression of bona fide NC factors, such as Snail and FoxD3, thus driving the onset of NC specification defined by the expression of Tfap2, Id, Myc, Myb, SoxE, and Ets gene family members (Luo et al., 2003; Sato et al., 2005; Hong and Saint-Jeannet, 2007; Sauka-Spengler et al., 2007; Milet et al., 2013; Schock and LaBonne, 2020). The persisting expression of these TFs, as well as the downstream activation of cadherins, integrins, signaling receptors and metalloproteases, subsequently lead to epithelial-to-mesenchymal transition (EMT) and delamination of $\mathrm{NC}$ cells from the dorsal neural tube. Cranial $\mathrm{NC}(\mathrm{cNC})$ cells migrate via canonical, well-established pathways to their final destinations within the vertebrate embryo's head. Unlike the $\mathrm{cNC}$ that can give rise to ectomesenchymal derivatives (forming the cartilage, bones, and connective tissues of the craniofacial skeleton), non-cranial, more posterior NC (vagal, trunk, sacral) form mostly neuronal derivatives such as the sensory neurons and glia in the dorsal root ganglia, sympathetic ganglia and enteric nervous system. Although cranial and trunk NC express similar groups of early marker genes, some distinct TFs (e.g., Sox8, Tfap $2 \beta$, Ets 1 ) driving cranial vs. trunk NC identity have been described 
(Simoes-Costa and Bronner, 2016). Recent work suggested that elaborate NC-GRN was progressively established during the evolution of vertebrates with trunk-like circuits being in place first (Martik et al., 2019). However, detailed analysis and understanding of the trunk NC-GRN remains to be done.

The dawn of the -omics era has contributed significantly to the elaboration and refinement of existing GRNs. Coupled with an increasing catalog of sequenced genomes, genome-wide approaches heralded an explosion of exploratory studies that not only recapitulated previous knowledge but also increased the rate of identification of novel developmental players. In the avian NC, RNA-seq identified multiple genes not known previously to be expressed in the migratory cNC such as $L m o 4, R x r G$, Ltk, and Col9a3 (Simões-Costa et al., 2014). Furthermore, work to compare the transcriptomes of trunk and $\mathrm{cNC}$ populations coupled with subsequent functional assays led to identification of a cranial-specific module in the migratory cNC consisting of Brn3c, Lhx5, Dmbx1 in the neural plate border; Sox8, Tfap $2 \beta$ in premigratory NC; and Ets1 (activated by Tfap $2 \beta$ ) (Simoes-Costa and Bronner, 2016). Overexpression of these factors in the trunk NC resulted in reprogramming of their identity, highlighted by the ectopic activity of a cNC-specific enhancer SOX10E2 (Betancur et al., 2010) and increased expression of chondrocyterelated genes Runx2 and Alx1. Importantly, gene modules are not limited to the "gross" distinction between trunk vs. cNC, as key differences in the molecular signature between $\mathrm{cNC}$ cells from different axial-levels could also be detected using RNA-seq (Lumb et al., 2017). Altogether, these studies exemplify the amenability of the cNC-GRN to be interrogated via an -omics-type approach for the desired outcome of identifying gene modules specific to subpopulations within the cNC.

Such efforts to resolve spatiotemporal dynamics of the cNC-GRN were further strengthened by emerging single cell technologies. Single cell RNA-seq (scRNA-seq) of $406 \mathrm{cNC}$ cells isolated from the avian embryo identified a fraction of invasive front cells (Trailblazers) with a distinct molecular signature that persisted through migration, therefore bringing to light subpopulations within one cell type with seemingly similar cell behavior (Morrison et al., 2017). Strikingly, scRNA-seq analysis of 1345 murine $\mathrm{CNC}$ cells detected a subtle but observable discrete cell state where cNC cells displayed a bias toward neuronal vs. mesenchymal fate during delamination from the neural tube (Soldatov et al., 2019). This finding refined and elaborated the long-held model of sequential (induction, specification, delamination, differentiation) developmental events underlying the cNC-GRN and demonstrated the fluid nature of $\mathrm{cNC}$ ontogeny at the transcriptional level. Pertinently, scRNAseq addressed a constantly debated question within the field concerning hetero- or homogeneity of premigratory NC cells. Investigation of transcriptional heterogeneity of premigratory NC cells in vivo using scRNA-seq of FAC-sorted foxd3-positive cNC cells from 5 to 6 ss zebrafish embryos (Lukoseviciute et al., 2018) failed to identify multiple specific NC subpopulations but singled-out a small cluster of NC cells which expressed low levels of factors key to NC specification-zic $2 b, \operatorname{tfap} 2 a$, sox10, twist $1 b$, ets 1, pax $3 a$, including foxd3. These cells expressed high levels of stem-cell state ("stemness") factors such as snaila, vent, vox, and $c x 43.4$, suggesting that they may represent non-specified $\mathrm{cNC}$ progenitors maintained in premigratory $\mathrm{cNC}$. This finding echoes an observation made by machine learning-based image analysis that clustered cNC cells based on expression of a selected panel of genes (including pluripotency and NC markers) within similar-staged avian embryos (Lignell et al., 2017).

From a GRN perspective, scRNA-seq called into question the existence of one unifying NC-GRN or multiple NC-GRNs working in concert with each other to drive NC development. Previous iterations of the NC-GRN were largely based on candidate gene approach studies, thus representing a summation of parts averaged across the $\mathrm{NC}$ as a whole. ScRNA-seq dissected this "unified" NC-GRN model into their parts, by revealing subpopulations with distinct molecular signatures (even if they were pre-enriched for $\mathrm{cNC}$ ) hinting at "multiple" NC-GRNs. In particular, comprehensive analysis of $\mathrm{NC}$ enhancer modules in the cranial region suggested that NC gene regulatory circuits controlling neuronal derivatives are established much earlier in the embryo and use non-exclusive cis-regulatory elements shared with neural programmes. In contrast, regulatory circuits underlying mesenchymal/canonical NC gene expression are laid down later when neural tissue is already defined. These later circuits use an intermediary cohort of enhancers active exclusively in the NC (Williams et al., 2019). Such dichotomy in regulatory element modules and $\mathrm{NC}$ circuits was also uncovered in the vagal NC giving rise to the enteric nervous system. The neuronal derivative programme was pleiotropic, whereas the GRN underlying neural/glial/mesenchymal derivatives was newly established and utilized by NC cells only (Ling and SaukaSpengler, 2019).

Methodologically, "first-generation" scRNA-seq studies prior to Williams et al. (2019) utilized FAC-sorting followed by sequencing of full-length mRNA transcripts on a relatively small number of single cells, an approach that although robust, was also laborious and limited in statistical power for sensitive clustering of subpopulations of cells. Nonetheless, they played an important role in priming the NC field for droplet-based technologies allowing a significantly higher number of cells (by the thousands, not hundreds) to be profiled at any one time, therefore bypassing this limitation. The powerful use of the latter approach was also demonstrated in the proto-vertebrate Ciona intestinalis, where the resolution achieved enabled identification of an ancestral Six, $M s x$, and Pax regulatory module shared between cranial placodes and NC in vertebrates (Horie et al., 2018).

\section{CIS-REGULATORY ELEMENTS UNIFY NC GENE MODULES}

Positive cis-regulatory elements, also known as enhancers, serve as important "switches" within GRN modules by integrating inputs/binding of upstream factors in order to coordinate output/expression of downstream targets. SOX10E1 and SOX10E2 enhancers, situated $1 \mathrm{~kb}$ downstream of the coding region for NC master regulator Sox10 (Kelsh, 2006; SaukaSpengler and Bronner-Fraser, 2008; Schock and LaBonne, 2020) have been shown to control the expression of Sox 10 in the chicken 
embryo (Betancur et al., 2010). Both enhancers demonstrated distinct spatiotemporal activity, where SOX10E2 alone was active in early delaminating cNC cells. Mutations at key binding motifs identified in SOX10E2, knockdown of upstream TFs and chromatin immunoprecipitation (ChIP) experiments confirmed Sox9, Ets1, and cMyb proteins as transcriptional inputs for endogenous Sox10 gene expression. Similarly, two enhancers, $\mathrm{NC} 1$ and $\mathrm{NC} 2$, located 20 and $44 \mathrm{~kb}$ upstream of the NC specifier FoxD3 have been shown to control FoxD3 gene expression in the avian embryo (Simões-Costa et al., 2012). NC1 (but not NC2) was found to be active in the premigratory $\mathrm{cNC}$, but its activity diminished during migration and no activity could be detected caudal to rhombomere 3. Knockdown of upstream factors such as Pax7, Msx1, Ets1, and Zic1 confirmed their participation in the FoxD3 module underlying gene regulation between trunk and cNC-Ets1 demonstrated cranial-specific control of NC1, Zic1 controlled vagal- and trunk-specific activity of NC2, while Pax7 and Msx1 inputs where shared between NC1 and NC2. Altogether, these case studies presented clear evidence for the role of enhancers in maintaining spatiotemporal expression of developmentally-regulated cNC genes. They spearheaded higher throughput genome-wide characterization of the global cNC landscape using approaches such as ChIP-seq (Barski et al., 2007) and ATAC-seq (Buenrostro et al., 2013) to profile large cohorts of NC enhancers both in the embryo and in vitro.

It has been shown that developmental enhancers display specific histone signatures, such as $\mathrm{H} 3 \mathrm{~K} 27 \mathrm{ac}$ and $\mathrm{H} 3 \mathrm{~K} 4 \mathrm{me} 1$, indicative of their active vs. poised chromatin states (Creyghton et al., 2010). Furthermore, several studies have demonstrated that chromatin remodelers and their associated histone marks are regulated during NC development (reviewed by StroblMazzulla et al., 2012). Large-scale epigenomic mapping using p300, H3K27Ac, H3K4me1, and H3K4me3 enrichment profiles successfully facilitated the identification of enhancer elements in human $\mathrm{cNC}$ cell culture, uncovering the association of a key NC specifier, TFAP2A, with permissive chromatin landscape at putative NC enhancers (Rada-Iglesias et al., 2012). This coupling between epigenetic modulation of enhancers and function was further strengthened by studies in mouse embryonic stem cells (mESC) elucidating the mechanism by which another NC specifier, FOXD3, acted to decommission enhancers via recruitment of specific chromatin remodelers (Krishnakumar et al., 2016; Respuela et al., 2016). In vivo, epigenome profiling of subpopulations of mouse cNC cells exposed the differences in chromatin signature reflective of their positional identity (Minoux et al., 2017).

\section{MULTIOMICS AND REBUILDING THE CNC-GRN}

The cNC-GRN has benefited from the substantial body of in vivo data, occasionally complemented by in vitro studies, from numerous labs over the past few decades. Pre-omics, a glaring knowledge gap persisted as experimental limitations lacked detail on the extent of the inter-connectivity between gene modules. Taking full advantage of the multiomics revolution and the demonstration of its utility in proof-of-principle characterization of early human embryos (Li et al., 2018), a multiomics approach was employed in multiple model organisms to re-examine the cNC-GRN. These studies sought to parse substantial biological information obtained from multiple levels: the NC genome (regions of open chromatin), transcriptome (RNA transcripts, including nascent transcripts), epigenome (chromatin modifications, chromatin-looping), and interactome (protein-DNA or protein-protein interactions) into workable hypotheses to test novel mechanisms, gene modules and players (Figure 1). For instance, omics interrogation of chromatin accessibility and looping during cNC development, in combination with transcriptional dynamics analyzed at both population and single-cell level in chick revealed a rich tapestry of gene modules. This not only provided insight into subcircuits underlying $\mathrm{cNC}$ heterogeneity (with identification of some novel inputs) but also enabled reverse engineering of gene regulatory circuits for every gene expressed, thus facilitating reconstruction of the global NC-GRN with unrivaled resolution (Williams et al., 2019). Combined with gold standard molecular techniques in the embryo such as enhancer screens and knockout experiments (Hockman et al., 2019; Ling and Sauka-Spengler, 2019; Williams et al., 2019), the collective result yielded as powerful resources with the potential to not only recapitulate previous work but also significantly expand on them. Ultimately, these studies accelerate progress for the myriad of biological questions-of-interest within the NC research community with far-reaching implications in biology, evolution, health and disease. Here, we briefly highlight recent findings in cNC-GRN biology resulting from multiomics.

\subsection{Molecular Mechanism of cNC Pioneer Factors}

FOXD3 transcription factor is an important player in the NCGRN (Lister et al., 2006; Montero-Balaguer et al., 2006; Stewart et al., 2006; Wang et al., 2011c) with evidence in embryonic stem cells documenting its possible cellular function as both a repressor and activator (Pohl and Knöchel, 2001; Yaklichkin et al., 2007; Krishnakumar et al., 2016; Respuela et al., 2016). A transgenic zebrafish line where the foxd3 locus has been disrupted with a Citrine or mCherry fluorophore (Hochgreb-Hägele and Bronner, 2013) was used to characterize FoxD3 bimodal properties within its native context in a developing embryo. By performing genetic crosses between foxd3-mCherry and foxd3Citrine heterozygote parents, foxd3-Citrine heterozygote and foxd3-mCherry/Citrine homozygote knockout NC cells were isolated by FACS for downstream multiomics analysis. Using a combination of RNA-seq, ATAC-seq and H3K27ac ChIP-seq, the NC transcriptome and epigenomic landscape were characterized across four embryonic stages key to $\mathrm{cNC}$ development within the context of the foxd3-DNA binding landscape (Lukoseviciute et al., 2018). Foxd3 was shown to prime NC gene expression in early pre-migratory $\mathrm{cNC}$ by binding to its target enhancers. Conversely, later in cNC development, it represses active enhancers associated with mesenchymal/neuronal genes in line with previous in vitro data (Krishnakumar et al., 2016; Respuela et al., 2016). In short, using multiomics to characterize the 


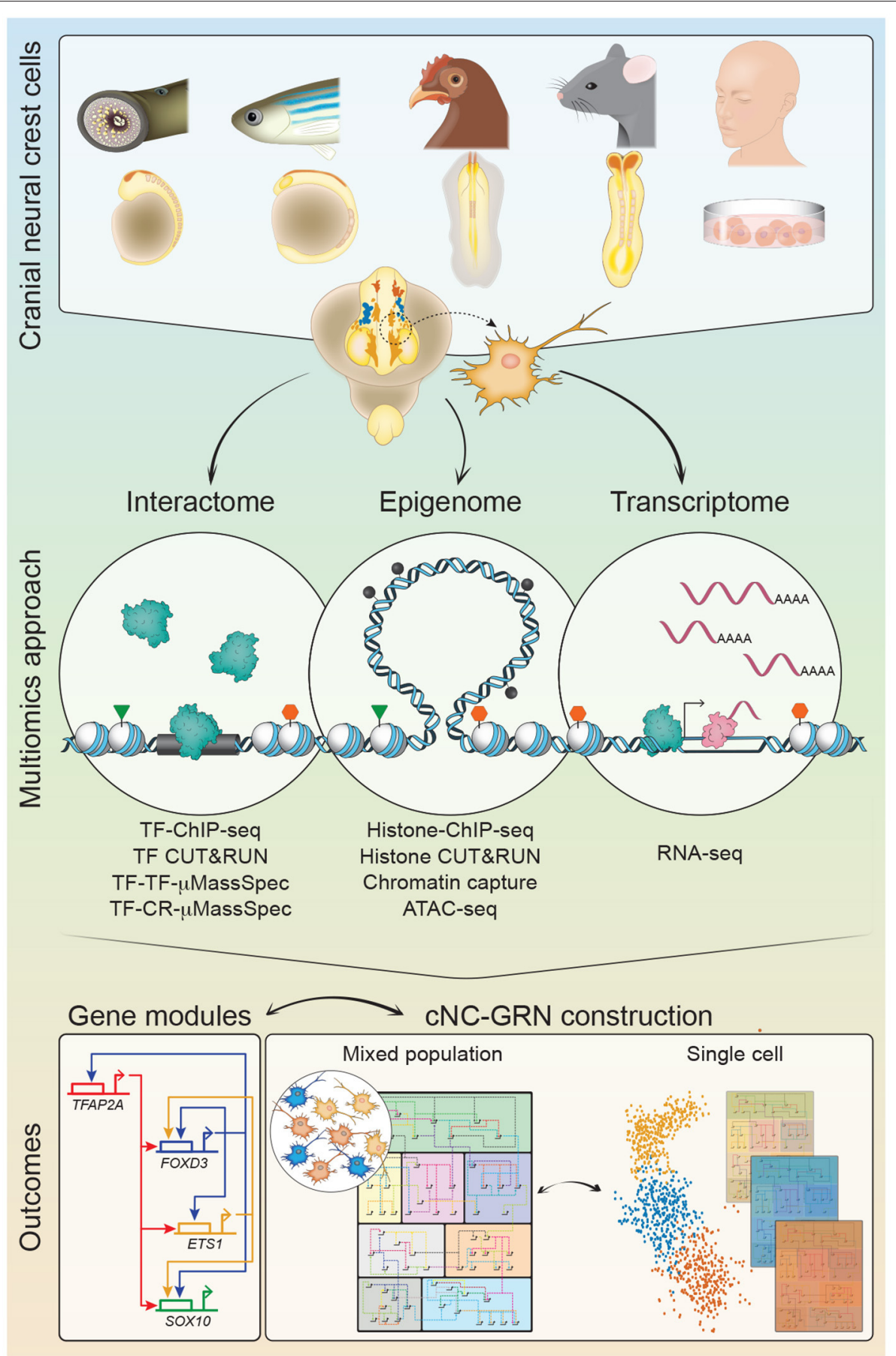

FIGURE 1 | A multiomics approach for construction of the cranial neural crest gene regulatory network (cNC-GRN). CNCCs from in vivo non-human embryo models and human pluripotent stem cell differentiation in vitro model were subjected to multiomics interrogation for global-level information. Interactome analyses resolve TF interactions to the genome (TF-ChIP-seq, TF CUT\&RUN), other TFs (TF-TF- $\mu$ MassSpec), or CRs (TF-CR- $\mu$ MassSpec). Epigenome analyses reveal enhancers and promoters defined by regions of accessible chromatin (ATAC-seq) and/or specific histone modifications (Histone-ChIP-seq, Histone CUT\&RUN). CUT\&RUN is an alternative method to ChIP-seq that has its utility demonstrated in the chick embryo NC (Skene and Henikoff, 2017; Rothstein and Simoes-Costa, 2020). Direct epigenomic relationships between promoters and enhancers are obtained by profiling their physical proximity (Chromatin capture). Transcriptome analysis provides snapshot of expressed genes. Parsing of all the datasets results in substantial number of gene modules to elaborate on the cNC-GRN, especially if coupled with single cell technologies for subpopulation resolution. CNCC, cranial neural crest cell; TF, transcription factor; CR, chromatin remodeler; $\mu$ MassSpec, micro mass spectrophotometry. 


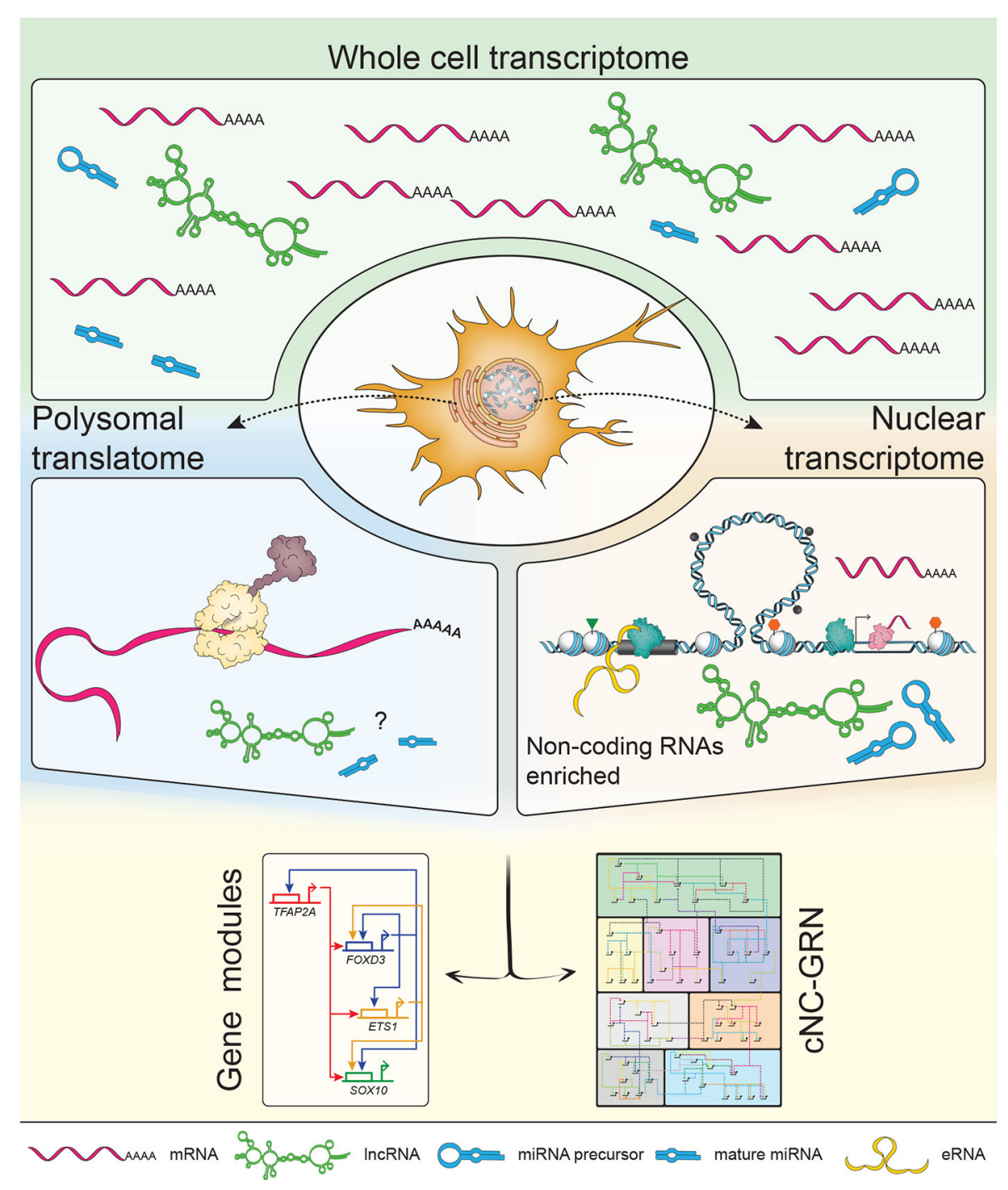

FIGURE 2 | Subcellular profiling increases resolution of the non-coding landscape. The transcriptome consists of a mixed population of protein-coding and non-coding RNAs, including but not limited to enhancer RNAs (eRNAs), long non-coding RNAs (IncRNAs) and microRNAs (miRNAs). Previous transcriptomic studies on populations of neural crest (NC) cells focused on polyadenylated mRNAs constituting mostly of protein-coding mRNAs. NC-specific subcellular profiling achieved by in vivo biotinylation of nuclei and ribosomes (i.e., polysomes) enables enrichment of RNA species subtypes already present in the whole cell transcriptome. The nuclear transcriptome provided higher definition of non-coding RNAs while the polysomal translatome minimized the "noise" of non-coding RNAs to inform on proteins being made (suggestive of dominant biological processes occurring at that stage). In depth exploration of non-coding RNAs' putative roles within the context of the cNC-GRN is currently underexplored but well-suited to the advantages provided by multiomics.

foxd3-GRN in vivo across cNC developmental stages revealed the transition between gene modules as foxd 3 shifted toward its canonical repressive activity after NC specification. This can be achieved by switching binding partners, a phenomenon that has been observed with another NC pioneer factor TFAP2A as it imposes its function in NC induction and specification modules by dimerising with TFAP2C or TFAP2B, respectively (Rothstein and Simoes-Costa, 2020).

\subsection{Vertebrate Evolution}

From an evolutionary perspective, NC enhancers are a distinct group of components within the cNC-GRN that are molded under evolutionary pressure leading to species divergence of craniofacial structures (Prescott et al., 2015). Due to their heavy contribution to the patterning of vertebrate craniofacial structures (reviewed in Santagati and Rijli, 2003), the cNC is of particular interest as a key contributor to the evolution of jawed vertebrates (Cerny et al., 2010). This is supported by candidate-based approach evidence in lamprey, a basal vertebrate, highlighting functional interactions between main components of the GRN underlying NC ontogeny (SaukaSpengler et al., 2007; Nikitina et al., 2008). Genome-wide studies in the lamprey were initially inhibited due to programmed largescale genome loss during embryonic development (Smith et al., 
2009), hampering acquisition of meaningful omics information despite the clear benefit for a more genome-wide approach as demonstrated in the basal chordate amphioxus (Yu et al., 2008). Publication of the lamprey germline genome (Smith et al., 2018) was a significant step forward in this regard and presented renewed opportunities to dissect the lamprey cNC-GRN using multiomics. By examining the transcriptional profiles of dorsal neural tube tissue containing the $\mathrm{cNC}$, modules that were both previously known in other vertebrates and unique to the lamprey were identified (Hockman et al., 2019). Concurrently, another study highlighted the resemblance of lamprey $\mathrm{cNC}$ to amniote trunk NC (Martik et al., 2019). Nevertheless, by additionally analyzing ATAC-seq profiles in dorsal neural tube tissue, novel cis-regulatory elements for two lamprey NC-GRN players$T f a p 2 B$ and SoxE1 were discovered. Strikingly, the lamprey SoxE1 enhancer was shown to be active in cNC-derived craniofacial features following integration into the zebrafish genome as well as in the amniote model, highlighting the potential for deep conservation of TF/enhancer interaction of NC-GRN enhancers (Hockman et al., 2019).

\section{FUTURE DIRECTIONS}

\subsection{Non-coding RNAs Provide an Additional Facet to the cNC-GRN}

RNAs derived from enhancers (enhancer RNAs or eRNAs) emerged following a study describing developmentally-regulated enhancers in mouse cortical neurons (Kim et al., 2010). Further studies demonstrated the sensitivity of eRNA induction as a hallmark of cellular response to biological stimuli (Wang et al., 2011a; Lam et al., 2013; Li et al., 2013), and suggested that eRNA transcription can be correlated to regulation of chromatin looping (Melo et al., 2013; Hsieh et al., 2014). While these in vitro studies painted an early picture of eRNA expression and their potential function in regulating enhancermediated gene expression, mechanistic details surrounding these observations remained elusive. Later studies attempted to address this conundrum by focusing on eRNA crosstalk with the chromatin landscape (Kaikkonen et al., 2013; Mousavi et al., 2013), eRNA potential function as molecular partners during gene regulation (Schaukowitch et al., 2014; Sigova et al., 2015), as well as attempted to distinguish between functionality of eRNA transcription or their RNA transcripts (Paralkar et al., 2016). Several recent studies have further shed light on eRNA transcription as a global indicator of activated gene expression programmes. Interrogation of the nuclear transcriptome of migrating $\mathrm{cNC}$ cells in zebrafish embryos detected bidirectional transcription at a global scale. This "feature" enabled clustering of putative enhancers that were also functionally associated with known NC genes (Trinh et al., 2017), in line with a previous report that suggested eRNA profiles were more indicative of enhancer activity compared to H3K27Ac ChIP-seq profiles (Zhu et al., 2013). A study by the FANTOM consortium further showed that genome-wide eRNA transcription appeared to be temporally regulated, often preceding transcription of associated protein-coding genes (Arner et al., 2015). In short, regardless of the biological function of eRNAs during development, their phenomenon in itself is able to highlight active regions of the non-coding genome. Therefore, characterization of eRNA transcriptomes has strong potential to inform on genome regulation mechanisms underlying the $\mathrm{CNC}-\mathrm{GRN}$.

Another class of under-explored non-coding RNA in development are long non-coding RNAs (lncRNAs). Landmark findings describing lncRNAs in the HOX locus, HOTAIR and HOTTIP, served as important case studies of modern lncRNA biology (Rinn et al., 2007; Tsai et al., 2010; Wang et al., 2011b). HOTAIR silenced gene expression at promoter regions of the HOXD locus by interacting with the chromatin remodeler Polycomb Repressive Complex 2 (PRC2) to facilitate H3K4 demethylation. Similar to the molecular mechanism of HOTAIR, HOTTIP from the HOXA locus was shown to recruit WDR5/MLL complexes and drive H3K4 trimethylation to activate gene transcription. Hence, coupling between lncRNA function and epigenetic regulation serves as a useful framework to address the roles of lncRNAs in GRNs underlying developmental programmes. It is also important to note that lncRNAs are not a completely novel discovery, as their presence at loci of imprinted genes were reported in the past. More recently, mechanisms of these "classical" IncRNAs were studied in detail. The "IncRNA-mediated chromatin regulation" model presented by HOTAIR and HOTTIP were echoed in studies involving Airn, H19, and Xist (Engreitz et al., 2013; Monnier et al., 2013; Santoro et al., 2013). Last but not least, RNA species from another class of non-coding RNA-microRNAs (miRNAs) - were also found to play roles in NC development with several candidates identified thus far (reviewed in Weiner, 2018). LncRNA and miRNA activities are not mutually exclusive and crosstalk between the two classes have been documented (Zheng et al., 2014; Tan et al., 2015). Altogether, the contribution of the non-coding genome serves as another exciting facet to development and evolution of the cNC-GRN-an uncharted territory ripe for exploration in the multiomics era.

\subsection{Compartmentalizing NC Molecular Identity}

Genome-wide profiling of polyadenylated transcripts from whole cell lysates provides a comprehensive snapshot of NC-GRN players being expressed at developmental stages-of-interest. Profiling polyadenylated transcripts alone, however, directly excludes non-polyadenylated RNAs enriched in the nucleus which form a large proportion of non-coding RNAs from intergenic regions (Carninci et al., 2005). This limitation can be addressed by subcellular profiling and rRNA-depletion during the construction of sequencing libraries. Isolating polysomes using recently-developed TRAP method (Heiman et al., 2014) and their associated mRNAs in the zebrafish migratory $\mathrm{NC}$ at 16-18 ss informed us of both known and novel NC markers forming the translatome at this developmental window (Chong, 2017). Enrichment of elavl3 suggested that at least a subset of these cells (i.e., actively migrating $\mathrm{cNC}$ and premigratory trunk $\mathrm{NC}$ ) were actively differentiating into their neuronal derivatives. On the other hand, by isolating nuclei transcriptomes at the 
same developmental stage, we demonstrated that functional annotation of transcribed enhancers (eRNAs) and promoters reflected the molecular signature of migratory NC cells and derivatives (Trinh et al., 2017); however the corresponding genes were not being translated as yet. Thus, similar to chromatin accessibility profiles which pre-defined cellular identities of cranial and vagal NC prior to associated gene expression (Ling and Sauka-Spengler, 2019; Williams et al., 2019), eRNA profiles also preceded coding gene transcription, thus reflecting future steps in NC ontogeny. Taken together, the translatome data provides a clearer picture without the "noise" from cytoplasmic or nuclear transcripts and suggested that at a given time-point, specification and/or differentiation of neuronal derivatives seem to dominate over ectodermal, mesodermal, and neuroepithelial derivatives (depleted in the translatome). These findings also highlight the utility of technologies to genetically attain subcellular resolution using in vivo biotinylation (de Boer et al., 2003; Deal and Henikoff, 2010; Trinh et al., 2018) with sufficient clarity to elucidate the role of non-coding RNAs in the cNC-GRN (Figure 2).

The contrast between the two pictures painted from nuclei and polysomes of NC cells at the same developmental stage raised important questions relating to our interpretation of how development proceeds at the cellular level. Traditionally, cellular identity has perhaps been defined over-simplistically via the expression of all protein markers in a GRN. We are now in the position to expand this definition by not only taking into account what protein(s) and where within the organism these players are involved, but also what non-coding element(s) are responsible for gene activation and where within a cell these new players are exerting their functions. Integrating this information is a next complex task on the agenda and is non-trivial given that NC cells transition from being a stem cell-like population to many subpopulations committed to different, not necessarily binary fates. It is therefore crucial to perform and integrate multiplex genetic lineage tracing analyses into this picture, interpret multiomics data at single cell and with subcellular resolution, as well as develop new, non-biased functional genomics integration tools based on artificial intelligence and deep learning approaches.

\section{DISCUSSION}

Embryology has progressed in leaps and bounds leading to the modern incarnation of developmental biology as we know it today. From embryological techniques to advances in genome biology, our understanding of animal development has reached impressive heights. Here, at the forefront of modern developmental genetics and genomics, we propose using a combination of "traditional" and "modern" methods to deepen our understanding of genetic programmes underlying cNC development encoded within the genome.

The $\mathrm{cNC}$ is a multipotent population of cells key to vertebrate evolution. It is a versatile system for interrogation, as the genetic machinery underlying its biology reiterates throughout development and disease. This well-oiled system is also sensitive to fine-tuned regulation; disrupt a cog and development fails to proceed normally leading to neurocristopathies that account for roughly $1 / 3$ of all birth defects. In order to discover ways to prevent or treat them, we first need to fully understand what the baseline scenarios are, at the level of genes within the context of a highly dynamic genome.

Tackling the non-coding genome has also uncovered noncoding RNA molecules that form part of the genetic regulation underlying cellular function. Previous work by many research groups has highlighted lncRNAs as molecular scaffolds that shuttle proteins to their target regions to regulate gene expression. eRNAs not only serve as "indicators" of when gene transcription onsets, but also have been proposed to facilitate chromosome-looping between enhancers and the promoters they regulate. Coupled with advances in gene editing including CRISPR/Cas, we are now in the position to design experiments with flexibility, efficiency and precision, from genome-wide screens of non-coding elements (Liu et al., 2016; Sanjana et al., 2016; Zhu et al., 2016) to in vivo decommissioning of NC enhancers for functional investigation (Williams et al., 2019).

In conclusion, we hope to not only propose fresh perspectives and potential avenues of investigation into the cNC-GRN but also challenge the reader to revisit how we study developmental biology as a whole. We are now ushering a new generation of scientists willing to embrace the exponential growth of molecular and computational tools at their disposal-the future is bright.

\section{DATA AVAILABILITY STATEMENT}

The original contributions presented in the study are included in the article/supplementary material, further inquiries can be directed to the corresponding author/s.

\section{AUTHOR CONTRIBUTIONS}

VC-M and TS-S contributed to the writing and editing of the manuscript. Both authors contributed to the article and approved the submitted version.

\section{FUNDING}

VC-M was funded by a Clarendon Fund doctorate scholarship. TS-S was funded by a Wellcome Trust Senior Research Fellowship (215615/Z/19/Z).

\section{ACKNOWLEDGMENTS}

In memory of José Luis Gómez-Skarmeta (1966-2020), a champion of multiomics, pioneering our efforts to understand genome regulation in development and evolution. We thank Véronique Juvin of SciArtWork for graphic design of the figures. 


\section{REFERENCES}

Arner, E., Daub, C. O., Vitting-Seerup, K., and Andersson, R. (2015). Transcribed enhancers lead waves of coordinated transcription in transitioning mammalian cells. Science 347, 1010-1015. doi: 10.1126/science. 1259418

Barski, A., Cuddapah, S., Cui, K., Roh, T. Y., Schones, D. E., Wang, Z., et al. (2007). High-resolution profiling of histone methylations in the human genome. Cell 129, 823-837. doi: 10.1016/j.cell.2007.05.009

Betancur, P., Bronner-Fraser, M., and Sauka-Spengler, T. (2010). Genomic code for Sox10 activation reveals a key regulatory enhancer for cranial neural crest. Proc. Natl. Acad. Sci. U.S.A. 107, 3570-3575. doi: 10.1073/pnas.0906596107

Buenrostro, J. D., Giresi, P. G., Zaba, L. C., Chang, H. Y., and Greenleaf, W. J. (2013). Transposition of native chromatin for fast and sensitive epigenomic profiling of open chromatin, DNA-binding proteins and nucleosome position. Nat. Methods 10, 1213-1218. doi: 10.1038/nmeth.2688

Carninci, P., Kasukawa, T., Katayama, S., Gough, J., Frith, M. C., Maeda, N., et al. (2005). The transcriptional landscape of the mammalian genome. Science 309, 1559-1563. doi: 10.1126/science.1112014

Cerny, R., Cattell, M., Sauka-Spengler, T., Bronner-Fraser, M., Yu, F., and Medeiros, D. M. (2010). Evidence for the prepattern/cooption model of vertebrate jaw evolution. Proc. Natl. Acad. Sci. U.S.A. 107, 17262-17267. doi: 10.1073/pnas.1009304107

Chong, V. (2017). Biotagging, a genetically encoded toolkit in the zebrafish, reveals novel non-coding RNA players during neural crest and myocardium development (Ph.D. thesis). University of Oxford, Oxford, United Kingdom.

Creyghton, M. P., Cheng, A. W., Welstead, G. G., Kooistra, T., Carey, B. W., Steine, E. J., et al. (2010). Histone H3K27ac separates active from poised enhancers and predicts developmental state. Proc. Natl. Acad. Sci. U.S.A. 107, 21931-21936. doi: 10.1073/pnas.1016071107

de Boer, E., Rodriguez, P., Bonte, E., Krijgsveld, J., Katsantoni, E., Heck, A., et al. (2003). Efficient biotinylation and single-step purification of tagged transcription factors in mammalian cells and transgenic mice. Proc. Natl. Acad. Sci. U.S.A. 100, 7480-7485. doi: 10.1073/pnas. 1332608100

Deal, R. B., and Henikoff, S. (2010). A simple method for gene expression and chromatin profiling of individual cell types within a tissue. Dev. Cell 18, 1030-1040. doi: 10.1016/j.devcel.2010.05.013

Engreitz, J. M., Pandya-Jones, A., McDonel, P., Shishkin, A., Sirokman, K., Surka, C., et al. (2013). The Xist lncRNA exploits three-dimensional genome architecture to spread across the X chromosome. Science 341:1237973. doi: $10.1126 /$ science. 1237973

Heiman, M., Kulicke, R., Fenster, R. J., Greengard, P., and Heintz, N. (2014). Cell type-specific mRNA purification by translating ribosome affinity purification (TRAP). Nat. Protoc. 9, 1282-1291. doi: 10.1038/nprot.2014.085

Hochgreb-Hägele, T., and Bronner, M. E. (2013). A novel FoxD3 gene trap line reveals neural crest precursor movement and a role for FoxD3 in their specification. Dev. Biol. 374, 1-11. doi: 10.1016/j.ydbio.2012.11.035

Hockman, D., Chong-Morrison, V., Green, S. A., Gavriouchkina, D., CandidoFerreira, I., Ling, I. T., et al. (2019). A genome-wide assessment of the ancestral neural crest gene regulatory network. Nat. Commun. 10:4689. doi: $10.1038 /$ s41467-019-12687-4

Hong, C.-S., and Saint-Jeannet, J.-P. (2007). The activity of Pax3 and Zic1 regulates three distinct cell fates at the neural plate border. Mol. Biol. Cell 18, 2192-2202. doi: 10.1091/mbc.e06-11-1047

Horie, R., Hazbun, A., Chen, K., Cao, C., Levine, M., and Horie, T. (2018). Shared evolutionary origin of vertebrate neural crest and cranial placodes. Nature 560, 228-232. doi: 10.1038/s41586-018-0385-7

Hsieh, C.-L., Fei, T., Chen, Y., Li, T., Gao, Y., Wang, X., et al. (2014). Enhancer RNAs participate in androgen receptor-driven looping that selectively enhances gene activation. Proc. Natl. Acad. Sci. U.S.A. 111, 7319-7324. doi: 10.1073/pnas.1324151111

Ikeya, M., Lee, S. M. K., Johnson, J. E., McMahon, A. P., and Takada, S. (1997). Wnt signalling required for expansion of neural crest and CNS progenitors. Nature 389:6654. doi: $10.1038 / 40146$

Kaikkonen, M. U., Spann, N. J., Heinz, S., Romanoski, C. E., Allison, K., a., et al. (2013). Remodeling of the enhancer landscape during macrophage activation is coupled to enhancer transcription. Mol. Cell 51, 310-325. doi: 10.1016/j.molcel.2013.07.010
Kelsh, R. N. (2006). Sorting out Sox10 functions in neural crest development. Bioessays 28, 788-798. doi: 10.1002/bies.20445

Kim, T.-K., Hemberg, M., Gray, J. M., Costa, A. M., Bear, D. M., Wu, J., et al. (2010). Widespread transcription at neuronal activity-regulated enhancers. Nature 465, 182-187. doi: 10.1038/nature09033

Krishnakumar, R., Chen, A. F., Pantovich, M. G., Danial, M., Parchem, R. J., Labosky, P. A., et al. (2016). FOXD3 regulates pluripotent stem cell potential by simultaneously initiating and repressing enhancer activity. Cell Stem Cell 18, 104-117. doi: 10.1016/j.stem.2015.10.003

LaBonne, C., and Bronner-Fraser, M. (1998). Neural crest induction in xenopus: evidence for a two-signal model. Development 125, 2403-2414.

Lam, M. T. Y., Cho, H., Lesch, H. P., Gosselin, D., Heinz, S., Tanaka-Oishi, Y., et al. (2013). Rev-Erbs repress macrophage gene expression by inhibiting enhancerdirected transcription. Nature 498, 511-515. doi: 10.1038/nature12209

Levine, M., and Davidson, E. H. (2005). Gene regulatory networks for development. Proc. Natl. Acad. Sci. U.S.A. 102, 4936-4942. doi: 10.1073/pnas.0408031102

Lewis, J. L., Bonner, J., Modrell, M., Ragland, J., Moon, R., Dorsky, R., et al. (2004). Reiterated Wnt signaling during zebrafish neural crest development. Development 131, 1299-1308. doi: 10.1242/dev.01007

Li, L., Guo, F., Gao, Y., Ren, Y., Yuan, P., Yan, L., et al. (2018). Single-cell multi-omics sequencing of human early embryos. Nat. Cell Biol. 20, 847-858. doi: 10.1038/s41556-018-0123-2

Li, W., Notani, D., Ma, Q., Tanasa, B., Nunez, E., Chen, A. Y., et al. (2013). Functional roles of enhancer RNAs for oestrogen-dependent transcriptional activation. Nature 498, 516-520. doi: 10.1038/nature12210

Lignell, A., Kerosuo, L., Streichan, S. J., Cai, L., and Bronner, M. E. (2017). Identification of a neural crest stem cell niche by Spatial Genomic Analysis. Nat. Commun. 8:1830. doi: 10.1038/s41467-017-01561-w

Ling, I. T., and Sauka-Spengler, T. (2019). Early chromatin shaping predetermines multipotent vagal neural crest into neural, neuronal and mesenchymal lineages. Nat. Cell Biol. 21, 1504-1517. doi: 10.1038/s41556-019-0428-9

Lister, J. A., Cooper, C., Nguyen, K., Modrell, M., Grant, K., and Raible, D. W. (2006). Zebrafish Foxd3 is required for development of a subset of neural crest derivatives. Dev. Biol. 290, 92-104. doi: 10.1016/j.ydbio.2005.11.014

Liu, S. J., Liu, S. J., Horlbeck, M. A., Cho, S. W., Birk, H. S., Malatesta, M., et al. (2016). CRISPRi-based genome-scale identification of functional long noncoding RNA loci in human cells. Science 355:aah7111. doi: 10.1126/science.aah7111

Lukoseviciute, M., Gavriouchkina, D., Williams, R. M., Hochgreb-Hagele, T., Senanayake, U., Chong-Morrison, V., et al. (2018). From pioneer to repressor: bimodal foxd3 activity dynamically remodels neural crest regulatory landscape in vivo. Dev. Cell 47, 608-628. doi: 10.1016/j.devcel.2018.11.009

Lumb, R., Buckberry, S., Secker, G., Lawrence, D., and Schwarz, Q. (2017). Transcriptome profiling reveals expression signatures of cranial neural crest cells arising from different axial levels. BMC Dev. Biol. 17:5. doi: 10.1186/s12861-017-0147-z

Luo, T., Lee, Y.-H., Saint-Jeannet, J.-P., and Sargent, T. D. (2003). Induction of neural crest in Xenopus by transcription factor AP2. Proc. Natl. Acad. Sci. U.S.A. 100, 532-537. doi: 10.1073/pnas.0237226100

Martik, M. L., Gandhi, S., Uy, B. R., Gillis, J. A., Green, S. A., Simoes-Costa, M., et al. (2019). Evolution of the new head by gradual acquisition of neural crest regulatory circuits. Nature 574, 675-678. doi: 10.1038/s41586-019-1691-4

Melo, C. A., Drost, J., Wijchers, P. J., van de Werken, H., de Wit, E., Vrielink, J., et al. (2013). ERNAs are required for p53-dependent enhancer activity and gene transcription. Mol. Cell 49, 524-535. doi: 10.1016/j.molcel.2012.11.021

Milet, C., Maczkowiak, F., Roche, D. D., and Monsoro-Burq, A. H. (2013). Pax3 and Zic1 drive induction and differentiation of multipotent, migratory, and functional neural crest in Xenopus embryos. Proc. Natl. Acad. Sci. U.S.A. 110, 5528-5533. doi: 10.1073/pnas.1219124110

Minoux, M., Holwerda, S., Vitobello, A., Kitazawa, T., Kohler, H., Stadler, M. B., et al. (2017). Gene bivalency at Polycomb domains regulates cranial neural crest positional identity. Science 355:6332. doi: 10.1126/science.aal2913

Monnier, P., Martinet, C., Pontis, J., Stancheva, I., Ait-Si-Ali, S., and Dandolo, L. (2013). H19 lncRNA controls gene expression of the Imprinted Gene Network by recruiting MBD1. Proc. Natl. Acad. Sci. U.S.A. 110, 20693-20698. doi: $10.1073 /$ pnas. 1310201110 
Monsoro-Burq, A.-H., Fletcher, R., and Harland, R. (2003). Neural crest induction by paraxial mesoderm in Xenopus embryos requires FGF signals. Development 130, 3111-3124. doi: 10.1242/dev.00531

Monsoro-Burq, A.-H., Wang, E., and Harland, R. (2005). Msx1 and Pax3 cooperate to mediate FGF8 and WNT signals during Xenopus neural crest induction. Dev. Cell 8, 167-178. doi: 10.1016/j.devcel.2004.12.017

Montero-Balaguer, M., Lang, M. R., Sachdev, S. W., Knappmeyer, C., Stewart, R. A., De La Guardia, A., et al. (2006). The mother superior mutation ablates foxd3 activity in neural crest progenitor cells and depletes neural crest derivatives in zebrafish. Dev. Dyn. 235, 3199-3212. doi: 10.1002/dvdy.20959

Morrison, J. A., Mclennan, R., Wolfe, L. A., Gogol, M. M., Meier, S., McKinney, M. C., et al. (2017). Single-cell transcriptome analysis of avian neural crest migration reveals signatures of invasion and molecular transitions. eLife 6:e28415. doi: 10.7554/eLife.28415

Mousavi, K., Zare, H., Dell'Orso, S., Grontved, L., Gutierrez-Cruz, G., Derfoul, A., et al. (2013). ERNAs promote transcription by establishing chromatin accessibility at defined genomic loci. Mol. Cell 51, 606-617. doi: 10.1016/j.molcel.2013.07.022

Nikitina, N., Sauka-Spengler, T., and Bronner-Fraser, M. (2008). Dissecting early regulatory relationships in the lamprey neural crest gene network. Proc. Natl. Acad. Sci. U.S.A. 105, 20083-20088. doi: 10.1073/pnas.0806009105

Paralkar, V. R., Taborda, C. C., Huang, P., Yao, Y., Kossenkov, A. V., Prasad, R., et al. (2016). Unlinking an IncRNA from its associated cis element. Mol. Cell 62, 104-110. doi: 10.1016/j.molcel.2016.02.029

Pohl, B. S., and Knöchel, W. (2001). Overexpression of the transcriptional repressor FoxD3 prevents neural crest formation in Xenopus embryos. Mech. Dev. 103, 93-106. doi: 10.1016/S0925-4773(01)00334-3

Prescott, S. L., Srinivasan, R., Marchetto, M. C., Grishina, I., Narvaiza, I., Selleri, L., et al. (2015). Enhancer divergence and cis-regulatory evolution in the human and chimp neural crest. Cell 163, 68-84. doi: 10.1016/j.cell.2015.08.036

Rada-Iglesias, A., Bajpai, R., Prescott, S., Brugmann, S. A., Swigut, T., and Wysocka, J. (2012). Epigenomic annotation of enhancers predicts transcriptional regulators of human neural crest. Cell Stem Cell 11, 633-648. doi: 10.1016/j.stem.2012.07.006

Respuela, P., Nikolić, M., Tan, M., Frommolt, P., Zhao, Y., Wysocka, J., et al. (2016). Foxd3 promotes exit from naive pluripotency through enhancer decommissioning and inhibits germline specification. Cell Stem Cell 18, 118-133. doi: 10.1016/j.stem.2015.09.010

Rinn, J. L., Kertesz, M., Wang, J. K., Squazzo, S. L., Xu, X., Brugmann, S., et al. (2007). Functional demarcation of active and silent chromatin domains in human HOX loci by noncoding RNAs. Cell 129, 1311-1323. doi: 10.1016/j.cell.2007.05.022

Rothstein, M., and Simoes-Costa, M. (2020). Heterodimerization of TFAP2 pioneer factors drives epigenomic remodeling during neural crest specification. Genome Res. 30, 35-48. doi: 10.1101/gr.249680.119

Sanjana, N., Wright, J., Zheng, K., Shalem, O., Fontanillas, P., Joung, J., et al. (2016). High-resolution interrogation of functional elements in the noncoding genome. Science 353, 1545-1549. doi: 10.1126/science.aaf7613

Santagati, F., and Rijli, F. M. (2003). Cranial neural crest and the building of the vertebrate head. Nat. Rev. Neurosci. 4, 806-818. doi: 10.1038/nrn1221

Santoro, F., Mayer, D., Klement, R. M., Warczok, K. E., Stukalov, A., Barlow, D. P., et al. (2013). Imprinted Igf2r silencing depends on continuous Airn lncRNA expression and is not restricted to a developmental window. Development 140, 1184-1195. doi: 10.1242/dev.088849

Sato, T., Sasai, N., and Sasai, Y. (2005). Neural crest determination by co-activation of Pax3 and Zic1 genes in Xenopus ectoderm. Development 132, 2355-2363. doi: $10.1242 /$ dev.01823

Sauka-Spengler, T., and Bronner-Fraser, M. (2008). A gene regulatory network orchestrates neural crest formation. Nat. Rev. Mol. Cell Biol. 9, 557-568. doi: $10.1038 / \mathrm{nrm} 2428$

Sauka-Spengler, T., Meulemans, D., Jones, M., and Bronner-Fraser, M. (2007). Ancient evolutionary origin of the neural crest gene regulatory network. Dev. Cell 13, 405-420. doi: 10.1016/j.devcel.2007.08.005

Schaukowitch, K., Joo, J.-Y., Liu, X., Watts, J. K., Martinez, C., and Kim, T.-K. (2014). Enhancer RNA facilitates NELF release from immediate early genes. Mol. Cell 56, 29-42. doi: 10.1016/j.molcel.2014.08.023

Schock, E. N., and LaBonne, C. (2020). Sorting sox: diverse roles for sox transcription factors during neural crest and craniofacial development. Front. Physiol. 11:1564. doi: 10.3389/fphys.2020. 606889

Schumacher, J. A., Hashiguchi, M., Nguyen, V. H., and Mullins, M. C. (2011). An intermediate level of BMP signaling directly specifies cranial neural crest progenitor cells in zebrafish. PLOS ONE 6:e27403. doi: 10.1371/journal.pone.0027403

Sigova, A., Abraham, B., Ji, X., Molinie, B., Hannett, N., Guo, Y. E., et al. (2015). Transcription factor trapping by RNA in gene regulatory elements. Science 350, 978-982. doi: 10.1126/science.aad3346

Simões-Costa, M., and Bronner, M. E. (2015). Establishing neural crest identity: a gene regulatory recipe. Development 142, 242-257. doi: 10.1242/dev. 105445

Simoes-Costa, M., and Bronner, M. E. (2016). Reprogramming of avian neural crest axial identity and cell fate. Science 352, 1570-1573. doi: 10.1126/science.aaf2729

Simões-Costa, M., Tan-Cabugao, J., Antoshechkin, I., Sauka-Spengler, T., and Bronner, M. E. (2014). Transcriptome analysis reveals novel players in the cranial neural crest gene regulatory network. Genome Res. 24, 281-290. doi: 10.1101/gr.161182.113

Simões-Costa, M. S., McKeown, S. J., Tan-Cabugao, J., Sauka-Spengler, T., and Bronner, M. E. (2012). Dynamic and differential regulation of stem cell factor FoxD3 in the neural crest is encrypted in the genome. PLoS Genet. 8:e1003142. doi: 10.1371/journal.pgen.1003142

Skene, P. J., and Henikoff, S. (2017). An efficient targeted nuclease strategy for high-resolution mapping of DNA binding sites. elife 6:e21856. doi: 10.7554/eLife. 21856

Smith, J. J., Antonacci, F., Eichler, E. E., and Amemiya, C. T. (2009). Programmed loss of millions of base pairs from a vertebrate genome. Proc. Natl. Acad. Sci. U.S.A. 106, 11212-11217. doi: 10.1073/pnas.0902358106

Smith, J. J., Timoshevskaya, N., Ye, C., Holt, C., Keinath, M. C., Parker, H. J., et al (2018). The sea lamprey germline genome provides insights into programmed genome rearrangement and vertebrate evolution. Nat. Genet. 50, 270-277. doi: 10.1038/s41588-017-0036-1

Soldatov, R., Kaucka, M., Kastriti, M. E., Petersen, J., Chontorotzea, T., Englmaier, L., et al. (2019). Spatiotemporal structure of cell fate decisions in murine neural crest. Science 364:6444. doi: 10.1126/science.aas9536

Stewart, R. A., Arduini, B. L., Berghmans, S., George, R. E., Kanki, J. P., Henion, P. D., et al. (2006). Zebrafish foxd3 is selectively required for neural crest specification, migration and survival. Dev. Biol. 292, 174-188. doi: 10.1016/j.ydbio.2005.12.035

Strobl-Mazzulla, P. H., Marini, M., and Buzzi, A. (2012). Epigenetic landscape and miRNA involvement during neural crest development. Dev. Dyn. 241, 1849-1856. doi: 10.1002/dvdy.23868

Tan, J. Y., Sirey, T., Honti, F., Graham, B., Piovesan, A., Merkenschlager, M., et al. (2015). Extensive microRNA-mediated crosstalk between lncRNAs and mRNAs in mouse embryonic stem cells. Genome Res. 25, 655-666. doi: 10.1101/gr.181974.114

Trinh, L. A., Chong-Morrison, V., Gavriouchkina, D., Hochgreb-Hägele, T., Senanayake, U., Fraser, S. E., et al. (2017). Biotagging of specific cell populations in zebrafish reveals gene regulatory logic encoded in the nuclear transcriptome. Cell Rep. 19, 425-440. doi: 10.1016/j.celrep.2017.03.045

Trinh, L. A., Chong-Morrison, V., and Sauka-Spengler, T. (2018). Biotagging, an in vivo biotinylation approach for cell-type specific subcellular profiling in zebrafish. Methods. 150, 24-31. doi: 10.1016/j.ymeth.2018.07.011

Tsai, M.-C., Manor, O., Wan, Y., Mosammaparast, N., Wang, J. K., Shi, Y., et al. (2010). Long noncoding RNA as modular scaffold of histone modification complexes. Science 329, 689-693. doi: 10.1126/science.1192002

Wang, D., Garcia-Bassets, I., Benner, C., Li, W., Su, X., Zhou, Y., et al. (2011a). Reprogramming transcription by distinct classes of enhancers functionally defined by eRNA. Nature 474, 390-394. doi: 10.1038/nature10006

Wang, K. C., Yang, Y. W., Liu, B., Sanyal, A., Corces-Zimmerman, R., Chen, Y., et al. (2011b). A long noncoding RNA maintains active chromatin to coordinate homeotic gene expression. Nature 472, 120-124. doi: 10.1038/nature09819

Wang, W. D., Melville, D. B., Montero-Balaguer, M., Hatzopoulos, A. K., and Knapik, E. W. (2011c). Tfap2a and Foxd3 regulate early steps in the development of the neural crest progenitor population. Dev. Biol. 360, 173-185. doi: 10.1016/j.ydbio.2011.09.019

Weiner, A. M. (2018). MicroRNAs and the neural crest: from induction to differentiation. Mech. Dev. 154, 98-106. doi: 10.1016/j.mod.2018.05.009 
Williams, R. M., Candido-Ferreira, I., Repapi, E., Gavriouchkina, D., Senanayake, U., Ling, I. T., et al. (2019). Reconstruction of the global neural crest gene regulatory network in vivo. Dev. Cell 51, 255-276. doi: 10.1016/j.devcel.2019.10.003

Yaklichkin, S., Steiner, A. B., Lu, Q., and Kessler, D. S. (2007). FoxD3 and Grg4 physically interact to repress transcription and induce mesoderm in Xenopus. J. Biol. Chem. 282, 2548-2557. doi: 10.1074/jbc.M607412200

Yu, J. K., Meulemans, D., McKeown, S. J., and Bronner-Fraser, M. (2008). Insights from the amphioxus genome on the origin of vertebrate neural crest. Genome Res. 18, 1127-1132. doi: 10.1101/gr.076208.108

Zheng, G. X. Y., Do, B. T., Webster, D. E., Khavari, P., a., and Chang, H. Y. (2014). Dicer-microRNA-Myc circuit promotes transcription of hundreds of long noncoding RNAs. Nat. Struct. Mol. Biol. 21, 585-590. doi: 10.1038/nsmb.2842

Zhu, S., Li, W., Liu, J., Chen, C.-H., Liao, Q., Xu, P., et al. (2016). Genome-scale deletion screening of human long non-coding RNAs using a paired-guide RNA CRISPR-Cas9 library. Nat. Biotechnol. 34, 1279-1286. doi: 10.1038/nbt.3715
Zhu, Y., Sun, L., Chen, Z., Whitaker, J. W., Wang, T., and Wang, W. (2013). Predicting enhancer transcription and activity from chromatin modifications. Nucleic Acids Res. 41, 10032-10043. doi: 10.1093/nar/ gkt826

Conflict of Interest: The authors declare that the research was conducted in the absence of any commercial or financial relationships that could be construed as a potential conflict of interest.

Copyright $\odot 2021$ Chong-Morrison and Sauka-Spengler. This is an open-access article distributed under the terms of the Creative Commons Attribution License (CC $B Y)$. The use, distribution or reproduction in other forums is permitted, provided the original author(s) and the copyright owner(s) are credited and that the original publication in this journal is cited, in accordance with accepted academic practice. No use, distribution or reproduction is permitted which does not comply with these terms. 